\title{
Neurobiologia das emoções
}

\author{
Neurobiology of emotions
}

Renato T. Ramos

Ramos RT. Neurobiologia das emoções / Neurobiology of emotions. Rev Med (São Paulo). 2015 set.-dez.;94(4):239-45.

RESUMO: Este artigo revê alguns conceitos gerais sobre os mecanismos biológicos envolvidos na expressão das emoções. O foco da discussão é a relevância destas informações para a formação do médico em geral. A hipótese de que comportamentos inatos e geneticamente controlados interagem fortemente com elementos sociais, culturais e familiares na gênese dos transtornos afetivos e ansiosos é discutida a partir de dados de estudos com modelos animais e estudos utilizando técnicas de neuroimagem funcional. Embora, muitas questões de caráter psicodinâmico e sociológico não sejam discutidas, o artigo chama a atenção para a importância, dentro da prática clínica, da articulação destes conhecimentos humanísticos com os modelos neurofisiológicos do comportamento humano.

Descritores: Emoções; Neurofisiologia; Transtornos mentais; Comportamento.

$\mathrm{O}$ comportamento humano é um fenômeno extremamente complexo e seu conhecimento é fundamental para a prática da medicina. Entender o papel das emoções na expressão destes comportamentos é particularmente importante devido ao papel dos estados afetivos na percepção e na modulação da interação do indivíduo com o mundo exterior. Por exemplo, existem evidências de que a sensação subjetiva de bem-estar é um fator essencial no desencadeamento e prognóstico de diversas condições clínicas ${ }^{1}$. Embora esta sensação dependa de condições materiais, relacionamentos sociais, familiares e de trabalho, não há dúvida de que estados
ABSTRACT: The article reviews some general concepts on the biological mechanisms of emotional expression. The focus is to discuss the potential relevance of these information for the clinical practice. The hypothesis that innate and genetically controlled behaviors strongly interact with social, cultural, and familiar factors in the genesis of mood and anxiety disorders is discussed based on data from studies involving animal models and functional neuroimaging techniques. Although not directly discussed, the article points out to the importance of psychodynamic and sociological determinants of human behavior.

Keywords: Emotions; Neurophysiology; Mental disorders; Behavior.

emocionais gerados interiormente e dependentes de fatores psicológicos e fisiológicos são seus principais determinantes. De fato, existem evidências de que a redução na percepção de bem-estar psicológico seja um fator de risco para o adoecimento como sugerido pela sua forte correlação com depressão, estresse, mortalidade prematura, doença coronariana, diabetes e outras doenças crônicas $^{2}$. De forma complementar, existem também evidências de que o aumento da sensação de bem-estar pode atuar também como um fator protetor contra o adoecimento ${ }^{3}$.

O estudo das emoções do ponto de vista

Professor Associado, Departamento de Psiquiatria, Faculdade de Medicina da Universidade de São Paulo. Laboratório de Psicofisiologia e Realidade Virtual (LIM-23). Instituto de Psiquiatria, HCFMUSP.

Endereço para correspondência. Rua Dr. Ovídio Pires de Campos, 785. CEP: 05403-010, Cerqueira César, São Paulo, SP. e-mail: rtramos@usp.br 
médico enfrenta o desafio de se construir um modelo fisiopatológico que integre os aspectos biológicos e psicossociais envolvidos na gênese do comportamento humano. Conceitos de natureza psicológica como personalidade, estresse social e neuroticismo precisam ser articulados com conceitos relativamente mais básicos como detecção de ameaças e comportamentos de defesa. De maneira semelhante, estes modelos devem explicar as relações entre os mecanismos neuronais e hormonais atuantes nos diferentes estados emocionais e funções cognitivas como atenção e memória, sempre levando em consideração os contextos nos quais as emoções se expressam.

Dentro deste quadro geral, este artigo visa rever alguns conceitos sobre os mecanismos biológicos das emoções potencialmente relevantes para a formação do médico em geral. Com isso, muitas questões de caráter psicodinâmico e sociológico não serão discutidas, apesar da clara importância destas abordagens para a prática clínica.

\section{A biologia do comportamento humano}

O comportamento humano como vemos atualmente é o resultado de um longo processo natural onde mecanismos biológicos evoluíram de forma a propiciar o surgimento de capacidades mentais complexas e, posteriormente, o desenvolvimento de sociedades e culturas. A hipótese mais aceita atualmente é a de que comportamentos mais simples associados a reações inatas, como reações de defesa, ainda façam parte do repertório de comportamentos humanos ${ }^{4}$. Porém, tais comportamentos relativamente mais primitivos tiveram que se moldar a novos contextos que envolvem valores sociais e morais.

Outra evidência a favor deste modelo evolutivo geral é a universalidade das formas de expressão emocional. Darwin ${ }^{5}$ já havia notado que expressões faciais, atitudes corporais, reações autonômicas e comportamentos instintivos eram semelhantes em diversas espécies, sugerindo um importante controle genético. A experiência emocional humana mostra-se também muito homogênea mesmo quando comparamos povos racial e geograficamente isolados e, além disso, as situações que normalmente evocam o medo e a ansiedade não são aleatórias ${ }^{4}$. Assim, parece fazer sentido se pensar que certas reações de medo que aumentassem as chances de sobrevivência no passado, como o medo de certos animais, ou de situações perigosas, como altura, ainda estejam presentes, como comportamentos de natureza "primitiva" mesmo em seres humanos.

Embora sejam descritas como pertencentes a um único grupo de fenômenos mentais, as emoções apresentam grande variedade em sua forma de expressão, no seu possível significado em termos comportamentais e principalmente no conjunto de estruturas cerebrais envolvidos em sua implementação.
Analisar as emoções humanas a partir deste enfoque evolutivo tem sido muito útil para o aperfeiçoamento dos modelos fisiopatológicos de transtornos mentais como depressão e ansiedade, além de servir de guia para o desenvolvimento de novos tipos de tratamento.

Um dos mais importantes modelos relacionando estruturas e função na mediação das emoções foi o modelo de sistema límbico, proposto por MacLean ${ }^{6}$. Implícito a este modelo estava a concepção de que cada variedade de afeto seria mediada por um grupo especializado de estruturas cerebrais que formariam um sistema integrado. Embora o conceito de sistema límbico continue presente nas concepções atuais, sua validade tem sido questionada tanto do ponto de vista estrutural quanto teórico ${ }^{7}$.

As dificuldades começam com o próprio conceito unitário de emoção. Por exemplo, estados emocionais aparentemente diversos como depressão e ansiedade, compartilham várias características como alterações nos processos de tomada de decisões, expressão de sintomas autonômicos e alterações da atividade atencional. Este compartilhamento de mecanismos fisiopatológicos pode explicar a alta comorbidade entre transtornos afetivos e ansiosos, talvez devido ao papel destas funções mentais no processo de captura de informações do meio ambiente e intepretação de contextos externos e internos.

Assim, mais do que propor a existência de estruturas especificas para a expressão de cada tipo de emoção, parece ser mais razoável tentar descrever como cada aspecto do funcionamento cognitivo é afetado por estados funcionais de forma mais global.

Um outro tipo de modelo biológico das emoções que ganhou evidência mais recentemente envolve a hipótese de lateralização da expressão afetiva. De acordo com este tipo de modelo, o hemisfério cerebral direito ocuparia um lugar de destaque na gênese das emoções ${ }^{8}$. Esta hipótese está baseada na observação de que lesões neste hemisfério, levam a um prejuízo no reconhecimento do conteúdo emocional de expressões faciais. No entanto, para alguns autores, atividade do hemisfério direito parece estar mais relacionada à percepção e expressão das emoções do que à experiência emocional propriamente dita. A experiência completa de um estado emocional dependeria muito mais do envolvimento de muitas outras regiões cerebrais (veja uma revisão destes conceitos em Murphy et al. ${ }^{8}$ ).

\section{Emoção e cognição}

Para o médico é também muito importante saber como as emoções afetam outras formas de comportamento. Por exemplo, para se avaliar e atuar sobre a atitude do paciente diante de doenças ou de problemas de outra natureza é importante saber como pensamentos, atitudes e decisões podem ser evocados e controlados. $\mathrm{O}$ entendimento de como os estados emocionais afetam esses comportamentos complexos tem demandado estudos 
focalizados tanto em funções mentais básicas quanto em comportamentos mais complexos, como o aprendizado.

Existem fortes evidências de que estados afetivos modulam de forma importante diversos aspectos da cognição, como atenção, percepção e memória. Atenção e percepção estão envolvidas nas primeiras etapas no processamento de informação e, portanto, influenciam também as fases subsequentes do processamento cognitivo como evocação e fixação de memórias, raciocínio e tomadas de decisão.

Estudos em laboratório envolvendo a livre exploração visual ou a busca voluntária por alvos em imagens mostradas em telas de computadores sugerem que indivíduos ansiosos apresentam um aumento da vigilância para estímulos potencialmente ameaçadores e uma dificuldade para desengajar a atenção destes estímulos em tarefas que demandam atenção voluntária. Em contraste, os indivíduos deprimidos não parecem apresentar este padrão de vigilância para ameaças em tarefas de atenção com visualização livre, mas apresentam redução na resposta de orientação a estímulos positivos, além de aumento da manutenção de olhar em estímulos de natureza depressiva. Estas observações sugerem a presença de um mecanismo conhecido como viés atencional que exerceria um papel importante na fisiopatologia da ansiedade e depressão. O conhecimento deste padrão enviesado de funcionamento cognitivo tem servido de base para o desenvolvimento de novas formas de psicoterapia cognitiva para alguns transtornos de ansiedade e depressão ${ }^{9,10}$.

A mobilidade visual como forma de se avaliar a alocação da atenção no espaço também tem sido usada para o estudo da influência das emoções sobre o funcionamento cognitivo. Sacadas, ou a direção do olhar para um certo objetivo e anti-sacadas, ou a direção do olhar na direção oposta de um certo estímulo, são exemplos de tipos de paradigmas experimentais utilizados. De forma geral, considera-se que o tempo necessário para que o indivíduo passe a efetuar sacadas ou anti-sacadas corretas em função dos estímulos apresentados reflita a eficiência do processamento cognitivo. Indivíduos ansiosos costumam apresentar melhor desempenho em tarefas que envolvam anti-sacadas, especialmente com estímulos de natureza ameaçadora, em contraste com estímulos positivos ou neutros ${ }^{11}$.

\section{Comportamentos inatos e aprendizado}

Um aspecto importante da fisiologia das emoções é que nem todos os componentes dos comportamentos afetivos precisam estar codificados de forma inata em estruturas cerebrais. De fato, parece que várias espécies animais apresentam um aprendizado facilitado para certos tipos de respostas. Marks ${ }^{12}$ resume estas características em dois conceitos. O conceito de "prepotency" indica que certos estímulos são especialmente notáveis para certa espécie, que os percebe e reconhece seletivamente entre outros estímulos, mesmo quando os encontra pela primeira vez. "Preparedness"13 é a ideia de que certos estímulos se associam mais facilmente com outros, evocando respostas específicas, por exemplo, diante de um sinal de perigo específico, cada espécie aprende certas respostas defensivas mais facilmente do que outras e o aprendizado é mais rápido na medida que estas respostas façam parte do repertório inato de reações de defesa.

O ser humano tem muito mais flexibilidade de reações do que qualquer outra espécie animal, mas suas possibilidades não são infinitas. A questão central não é se determinar se o medo ou a ansiedade são comportamentos totalmente inatos ou totalmente aprendidos, mas ter em mente que certos tipos de aprendizado ocorrem com maior facilidade.

Estes aspectos do aprendizado foram esquecidos por muito tempo e muitos teóricos consideravam que as leis do aprendizado seriam universais para todas as espécies, que todos os tipos de estímulos e de respostas seriam equipotenciais e que fobias seriam simples repostas condicionadas de medo ${ }^{4}$.

Um dos pioneiros na demonstração experimental destas características de aprendizado em seres humanos foram Öhman et al. ${ }^{14}$. Em seus experimentos, um grupo de voluntários é apresentado a grupos de imagens com estímulos usualmente ameaçadores (como cobras ou aranhas) e com estímulos neutros (como flores, cogumelos ou caixas). Os indivíduos eram apresentados aos estímulos isoladamente para se obter um padrão basal. Em seguida, na fase de "aquisição" alguns estímulos eram rapidamente seguidos de um estímulo desconfortável como um choque leve ou um ruído. A seguir, na fase de extinção, os estímulos eram novamente apresentados, mas sem serem seguidos de qualquer estímulo doloroso. Alterações na condutância da pele e frequência cardíaca foram medidas.

Os resultados mostraram que os estímulos ameaçadores (cobras, aranhas) mostravam uma resposta diferente, com reação de alerta mais intensa, já na primeira fase do experimento, sem qualquer associação com estímulos desagradáveis ("prepotency"). O condicionamento de respostas autonômicas ocorreu mais rapidamente quando o estímulo doloroso era associado a estes diapositivos do que com aqueles com estímulos neutros ("preparedness"). Finalmente, o condicionamento de reações autonômicas frente a estímulos ameaçadores requereu um tempo muito maior para a sua extinção.

A relação entre influências ambientais e genéticas também tem sido explorada em modelos animais de outras condições clínicas além da ansiedade e medo. Por exemplo, o modelo de derrota social crônica tem sido explorado como equivalente de estresse social capaz de produzir comportamentos associado à depressão, incluindo anedonia (perda de capacidade de sentir prazer), desamparo 
comportamental e prejuízos na interação social. Neste modelo, um rato intruso é colocado em contato com um rato residente agressivo por períodos de 5 a 10 minutos, diariamente, por cerca de dez dias. Entre esses períodos de exposição, o rato que sofreu a derrota social é alojado em frente ao agressor separado por uma grade que reforça o estresse social, via contato sensorial, mas não permite o contato físico ${ }^{15}$.

Ratos suscetíveis a este paradigma exibem uma resposta de medo condicionado exagerado associado a alterações autonômicas mais pronunciadas. Estas alterações fisiológicas parecem ser mediadas através de aumentos locais nos níveis de BDNF (Brain Derived Neurotrophic Factor) na região basolateral da amígdala, resposta esta que pode ser inibida pela administração sistêmica de um antagonista beta-adrenérgico ${ }^{16}$.

Além disso, o comportamento resiliente ou de tolerância a estas condições parece estar relacionado com um conjunto de alterações da atividade neuronal em regiões específicas do cérebro, tais como a sobre-regulação da deltaFosB no núcleo accumbens ou a sub-regulação da histona deacetilase (HDAC6) em neurônios serotoninérgicos na rafe dorsal. Estes mecanismos fisiológicos de regulação da atividade emocional podem sugerir novos modelos de exploração no desenvolvimento de novos tratamentos para a depressão $0^{15}$.

\section{Emoções e neuroimagem funcional}

Em anos recentes, o desenvolvimento de técnicas de neuroimagem funcional, em especial a Ressonância Nuclear Magnética Funcional (fMRI, na denominação inglesa) tem permitido uma série de estudos que parecem sugerir a existência de alguns substratos cerebrais mais bem definidos para as emoções de medo, nojo e raiva. Em contraste, estes mesmos estudos sugerem uma falta de evidências sobre uma ligação funcional específica entre estruturas cerebrais e a gênese de estados como felicidade e tristeza ${ }^{8}$. Esses estudos permitem um acesso único ao funcionamento do cérebro humano e permitem uma visão integrada da atividade de suas diferentes regiões.

\section{Medo e amígdala}

Estudos funcionais sugerem um importante papel da amígdala nas reações de medo embora outras regiões como córtex cingulado anterior e córtex órbitofrontal sejam também muito importantes. A atividade da amígdala parece ser específica para o medo e pouco presente em estados como nojo, raiva e felicidade. Alguns investigadores descrevem a função da amígdala como de um "detector de ameaças" inespecífico ${ }^{17}$. Em contraste, existe também a possibilidade de a amígdala não estar envolvida especificamente na detecção de ameaça mas sim a um despertar emocional mais geral ${ }^{18}$. Estudos funcionais enfrentam dificuldades para lidar com esta questão pois algumas emoções são difíceis de serem separadas de estados inespecíficos de excitação. No entanto, na raiva, que pode ser considerada uma emoção de alta excitação, a amígdala mostra-se ativa em uma proporção muito pequena dos estudos, sugerindo que esta esteja mais especificamente envolvida no processamento do medo do que no processamento do componente de excitação de estímulos emocionais.

Embora o papel da amígdala no condicionamento do medo esteja bem estabelecido, ela também parece estar envolvida numa vasta gama de outras funções relacionadas com a informação emocional. Por exemplo, existem evidências de que a amígdala também esteja envolvida na orientação da atenção espacial em direção a estímulos emocionalmente relevantes, tais como a exploração da região dos olhos diante de imagens de faces humanas exprimindo emoções. Porém, como o direcionamento da atenção a certas características básicas do estímulo apresentado geralmente precede a atenção espacial, é possível que a amígdala esteja envolvida mais na identificação da natureza dos estímulos do que na sua localização espacial ${ }^{19}$.

\section{Nojo: globo pálido e insula}

O nojo é outro estado emocional cujo componente biológico também parece ser preponderante e existem evidências de que o globo pálido e a ínsula sejam particularmente responsáveis por sua expressão. Numa meta-análise de estudos com ressonância magnética funcional, Murphy et al. relatam que a ínsula se mostrou ativa na maioria dos estudos que envolvem a provocação de nojo ou aversão. O globo pálido também se mostrou ativo em mais de $70 \%$ dos estudos de aversão, mas em menos de $25 \%$ dos estudos dirigidos a outras emoções ${ }^{8}$. As reações de nojo e aversão são particularmente relevantes para o estudo de alguns transtornos psiquiátricos.

Por exemplo, o transtorno obsessivo-compulsivo (TOC) é caracterizado por pensamentos obsessivos e comportamentos compulsivos. Pensamentos obsessivos são ideias, imagens ou impulsos que surgem na consciência de forma repetitiva e estereotipada, causando sofrimento ou repugnância por se referirem a temas violentos ou obscenos ou por serem percebidas como irracionais e contrárias à vontade do indivíduo. Compulsões são comportamentos repetitivos e intencionais executados sempre da mesma maneira. Tais comportamentos não tem um fim em si mesmos e procuram prevenir a ocorrência de eventos negativos, geralmente ligados aos conteúdos das obsessões $^{20}$. Estudos de neuro-imagem funcional sugerem que pacientes com TOC apresentam maior propensão a relatar nojo e aversão quando expostos a imagens repugnantes em comparação a indivíduos saudáveis ou com outras formas de ansiedade. No entanto, estes grupos 
de indivíduos não parecem apresentar diferenças entre si em relação as respostas autonômicas quando expostos aos estímulos aversivos. A tendência em responder com aversão em contextos não ligados ao nojo parece associarse mais à intensidade das crenças obsessivas, sugerindo um importante papel modulador do comportamentos obsessivo-compulsivo por parte de crenças ou mecanismos de aprendizado ${ }^{21}$.

Em outro exemplo clínico, pacientes com Doença de Huntington mostram um prejuízo importante na sua capacidade de reconhecer sinais de repulsa, em estudos utilizado imagens de expressões faciais como estímulo, sugerindo que os gânglios da base também estejam envolvidos na expressão de tais comportamentos ${ }^{22}$.

\section{Raiva e o córtex orbitofrontal}

Estudos da neurofisiologia da raiva utilizando fMRI tem encontrado evidências de uma maior ativação de regiões do córtex órbitofrontal lateral durante a evocação desta emoção. Lesões nestas regiões têm sido associadas a comportamentos agressivos em humanos e em outros primatas. Anormalidades no processamento de informações de natureza social (por exemplo, redução na percepção de expressões faciais) têm sido associadas a comportamentos agressivos. Trabalhando com vídeos de expressões faciais e utilizando fMRI, Beyer et al. ${ }^{23}$ observaram que a reatividade do córtex orbitofrontal medial para expressões faciais de raiva correlacionou-se negativamente com o comportamento agressivo enquanto a atividade no córtex cingulado anterior correlacionou-se positivamente com este comportamento em resposta a expressões de raiva. Além disso, os autores encontraram um efeito de expressões de raiva sobre os padrões de atividade neural durante fases posteriores da tarefa, sugerindo que o efeito destas expressões na reatividade neural é mais do que apenas uma resposta de curto prazo.

\section{Exemplo clínico: biologia da ansiedade}

Talvez a ansiedade e as fobias sejam o exemplo clínico mais direto da influência dos modelos biológicos no estudo de patologias mentais. Como dito inicialmente, considera-se atualmente que reações de medo, que seriam mais adaptativas em ambientes naturais, ainda se expressem de forma espontânea em seres humanos mesmo fora destes contextos.

Além destas evidências de que as reações de medo e ansiedade não são aleatórias em indivíduos normais, certas características do desenvolvimento de distúrbios fóbicos e ansiosos sugerem um padrão estereotipado de reações compatível com o exposto até agora. Tomemos como exemplo os ataques de pânico e sua relação com a agorafobia. $\mathrm{O}$ ataque de pânico é o sintoma central do transtorno de pânico, podendo ser descrito como crise de início súbito que deve atingir seu pico de intensidade em até 10 minutos, com uma duração total de cerca de 15 a 30 minutos. Uma sensação de morte, descontrole ou enlouquecimento iminente acompanhada de sintomas físicos como falta de ar, tonteira, balanço, flutuação, mãos úmidas, formigamentos, boca seca, náuseas, diarreia, urgência para urinar, ondas de calor e frio e palpitações. $\mathrm{O}$ medo e a ansiedade são crescentes, acompanhados da certeza de que algo estranho e muito grave está acontecendo. Pode ocorrer também uma sensação de despersonalização ou desrealização. A agorafobia acompanha os ataques de pânico na grande maioria dos casos e se caracteriza pelo comportamento de esquiva de diversas situações públicas, pelo receio de afastar-se de casa ou de pessoas que lhe forneçam segurança. A preocupação prevalente nestes pacientes é a da ocorrência de algum mal-estar ou descontrole em locais de onde sair seja difícil ou embaraçoso ${ }^{20}$.

Embora muitos pacientes relacionem o início de seus sintomas com certas situações de "estresse", muitos deles não conseguem ver razões objetivas para começarem a apresentar ataques de pânico. Como que vindo do nada, estes ataques tomam o paciente de surpresa, podendo ocorrer, em qualquer situação. Na maioria dos casos, estes ataques deixam de ser aleatórios e passam a ocorrer preferencialmente em situações agorafóbicas.

Em alguns casos, um padrão de extensa esquiva de situações como locais cheios, filas, transporte coletivo, é rapidamente desenvolvido mesmo que um ataque de pânico nunca tenha ocorrido em cada um destes lugares especificamente ${ }^{13}$. Estes dados sugerem que o aparecimento de ataques de pânico, por motivos ainda desconhecidos, leve ao desencadeamento de um padrão prédeterminado de comportamentos complexos, em grande parte incompreensíveis para o paciente.

Uma característica interessante dos ataques de pânico é a possibilidade de sua manipulação experimental. Já na década de 1960 observava-se que a infusão de lactato de sódio era capaz de provocar ataques agudos de pânico em cerca de dois terços dos pacientes da mesma forma que a inalação de uma mistura de dióxido de carbono (CO2) a $5 \%$. Estas observações levaram à hipótese de que os ataques de pânico poderiam estar relacionados à anormalidades em quimioreceptores que, no caso de ataques espontâneos, estariam sendo ativados inadequadamente ${ }^{24}$.

Estudos genéticos sugerem que fatores ambientais e herdados interajam na gênese tanto do transtorno de pânico quando da ansiedade de separação observada em crianças. Estas condições clínicas parecem compartilhar uma sensibilidade aumentada a inalação de CO2 como um endofenótipo comum. Esta sensibilidade, no entanto, não parece ser um fenômeno biológico simples. Estudos de laboratório sugerem que o aparecimento de uma maior sensibilidade a $\mathrm{CO} 2$ é mais comum em ratos que experimentaram a separação materna em fases iniciais 
da $\operatorname{vida}^{25}$. Esta observação sugere que a expressão de características fisiológicas herdadas dependa fortemente das condições ambientais enfrentadas em fases iniciais do desenvolvimento do indivíduo.

Para explicar o aparecimento dos ataques de pânico, Klein et al. ${ }^{24}$ sugerem a hipótese da existência de um sistema de alarme para sufocação como um monitor fisiológico para situações de asfixia e cujo resposta comportamental final seria a indução de uma premência de fuga do local onde o indivíduo estaria. Este comportamento de premência de fuga seria um dos marcos clínicos da agorafobia.

do aperfeiçoamento das técnicas de intervenção em psicoterapia para o adequado tratamento destes pacientes.

\section{REFERÊNCIAS}

1. Steptoe A, Deaton A, Stone AA. Subjective wellbeing, health, and ageing. Lancet. 2015;385(9968):640-8. doi: 10.1016/ S0140-6736(13)61489-0.

2. Chida Y, Steptoe A. Positive psychological well-being and mortality: a quantitative review of prospective observational studies. Psychosom Med. 2008;70(7):741-56. doi: 10.1097/ PSY.0b013e31818105ba.

3. Lyubomirsky S, King L, Diener E. The benefits of frequent positive affect: does happiness lead to success? Psychol Bull. 2005;131(6):803-55. http://dx.doi.org/10.1037/00332909.131.6.803.

4. Marks I. Phobias and related anxiety disorder. BMJ. 1991;302(6784):1037-8. Available from: http://www. ncbi.nlm.nih.gov/pmc/articles/PMC1669645/.

5. Darwin C. The expression of the emotions in man and animals. London: J. Murray; 1872.

6. MacLean PD. Psychosomatic disease and the visceral brain; recent developments bearing on the Papez theory of emotion. Psychosom Med. 1949;11(6):338-53.

7. LeDoux JE. Emotion circuits in the brain. Annu Rev Neurosci. 2000;23:155-84. doi: 10.1146/annurev.neuro.23.1.155

8. Murphy FC, Nimmo-Smith I, Lawrence AD. Functional neuroanatomy of emotions: a meta-analysis. Cogn Affect Behav Neurosci. 2003;3(3):207-33. doi: 10.3758/ CABN.3.3.207

9. Armstrong T, Olatunji BO. Eye tracking of attention in the affective disorders: a meta-analytic review and synthesis. Clin Psychol Rev. 2012;32(8):704-23.

10. Bar-Haim Y. Research review: attention bias modification (ABM): a novel treatment for anxiety disorders. J Child Psychol Psychiatry. 2010;51(8):859-70. doi: 10.1111/j.14697610.2010.02251.x.

11. Ansari TL, Derakshan N. The neural correlates of cognitive

\section{COMENTÁRIOS FINAIS}

Objetivo deste artigo foi discutir de forma resumida a importância dos modelos biológicos para o entendimento das emoções humanas. Além disso, buscou-se mostrar alguns exemplos para ilustrar a importância deste tipo de conhecimento para a prática médica. Saber que comportamentos inatos e geneticamente controlados interagem fortemente com elementos sociais, culturais e familiares na gênese dos transtornos afetivos e ansiosos é fundamental para que o clínico entenda a importância tanto do desenvolvimento de novos fármacos quanto

effort in anxiety: effects on processing efficiency. Biol Psychol. 2011;86(3):337-48. doi: 10.1016/j.biopsycho.2010.12.013.

12. Marks I, Horder J. Phobias and their management. Br Med J (Clin Res Ed). 1987;295(6598):589-91. doi: 10.1016/j. biopsycho.2010.12.013.

13. Seligman ME, Ives CE, Ames H, Mineka S. Conditioned drinking and its failure to extinguish: avoidance, preparedness, or functional autonomy? J Comp Physiol Psychol. 1970;71(3):411-9.

14. Ohman A, Mineka S. Fears, phobias, and preparedness: toward an evolved module of fear and fear learning. Psychol Rev. 2001;108(3):483-522. http://dx.doi.org/10.1037/0033295X.108.3.483

15. Krishnan V. Defeating the fear: new insights into the neurobiology of stress susceptibility. Exp Neurol. 2014;261:412-6. doi: 10.1016/j.expneurol.2014.05.012.

16. Chou D, Huang CC, Hsu KS. Brain-derived neurotrophic factor in the amygdala mediates susceptibility to fear conditioning. Exp Neurol. 2014;255:19-29. doi: 10.1016/j. expneurol.2014.02.016.

17. Mineka S, Ohman A. Phobias and preparedness: the selective, automatic, and encapsulated nature of fear. Biol Psychiatry. 2002;52(10):927-37. doi:10.1016/S0006-3223(02)01669-4.

18. Dejean C, Courtin J, Rozeske RR, Bonnet MC, Dousset V, Michelet T, et al. Neuronal circuits for fear expression and recovery: recent advances and potential therapeutic strategies. Biol Psychiatry. 2015. doi: 10.1016/j.biopsych.2015.03.017.

19. Jacobs RH, Renken R, Aleman A, Cornelissen FW. The amygdala, top-down effects, and selective attention to features. Neurosci Biobehav Rev. 2012;36(9):2069-84. doi: 10.1016/j.neubiorev.2012.05.011.

20. Ramos R. Transtornos de ansiedade. In: Lopes A, editor. Tratado de clínica médica. São Paulo: Rocca; 2006. v.2, p. 2480-90.

21. Whitton AE, Henry JD, Grisham JR. Cognitive and psychophysiological correlates of disgust in obsessive- 
compulsive disorder. Br J Clin Psychol. 2015;54(1):16-33. doi: 10.1111/bjc.12058.

22. Dogan I, Saß C, Mirzazade S, Kleiman A, Werner CJ, Pohl A, et al. Neural correlates of impaired emotion processing in manifest Huntington's disease. Soc Cogn Affect Neurosci. 2014;9(5):671-80. doi: 10.1093/scan/nst029.

23. Beyer F, Münte TF, Göttlich M, Krämer UM. Orbitofrontal cortex reactivity to angry facial expression in a social interaction correlates with aggressive behavior. Cereb Cortex. 2014. doi: 10.1093/cercor/bhu101.

24. Klein DF. Historical aspects of anxiety. Dialogues Clin Neurosci. 2002;4(3):295-304. Available from: http://www. dialogues-cns.com/publication/historical-aspects-of-anxiety/.

25. Battaglia M, Ogliari A, D’Amato F, Kinkead R. Early-life risk factors for panic and separation anxiety disorder: insights and outstanding questions arising from human and animal studies of CO2 sensitivity. Neurosci Biobehav Rev. 2014;46 pt 3:455-64. doi: 10.1016/j.neubiorev.2014.04.005. 